\title{
MODELOS ALOMÉTRICOS PARA ESTIMAR EL ALMACENAMIENTO DE CARBONO DE BOSQUES MONTANOS BAJOS EN EL SUR DEL ECUADOR
}

\author{
ALLOMETRIC MODELS TO ESTIMATE STOCKS CARBON IN THE LOWER MONTANE \\ FORESTS IN SOUTH OF ECUADOR
}

Deicy Carolina Lozano Sivisaca ${ }^{1}$ Byron Gonzalo Palacios Herrera ${ }^{2}$ Zhofre Huberto Aguirre Mendoza ${ }^{3}$

\section{RESUMEN}

En Ecuador existen bosques montanos bajos de gran importancia biológica que proveen de servicios ecosistémicos como el almacenamiento de carbono. Por esta razón, se desarrolló una investigación que permitió elaborar modelos alométricos mediante la cuantificación de carbono en el bosque montano bajo. Se estableció una parcela permanente de una hectárea y se midieron todos los árboles con diámetro a la altura del pecho $\left(\mathrm{DAP}_{1,30 \mathrm{~m}}\right) \geq 5 \mathrm{~cm}$. Las variables que se usaron para la generación de los modelos fueron DAP y altura total (ht). La biomasa arbórea fue de $322,37 \mathrm{t} \mathrm{ha}^{-1}$ y el carbono total estimado fue de 161,07 $\mathrm{t}$ $\mathrm{ha}^{-1}$. En los modelos alométricos del estrato arbóreo, las variables que mejor se ajustaron fueron DAP, DAP ${ }^{2}$ y ht. La familia botánica con mayor biomasa $\left(69,95 \mathrm{t} \mathrm{ha}^{-1}\right)$ y carbono $\left(34,98 \mathrm{t} \mathrm{C} \mathrm{ha}^{-1}\right)$ fue Moraceae, que conjuntamente con Lauraceae, Myristicaceae y Rubiaceae almacenan el 57\% de carbono $\left(91,55 \mathrm{t} \mathrm{C}^{-1}\right.$ ) del bosque montano bajo. De las 179 especies botánicas registradas, las especies Pseudolmedia laevigata, Nectandra reticulata, Otoba parvifolia, Vochysia paraensis, Ficus sp. y Elaeagia obovata almacenan el $51 \%\left(81 \mathrm{t} \mathrm{C} \mathrm{ha}^{-1}\right)$ del carbono total estimado.

Palabras claves: biomasa; servicio ecosistémico; Lauraceae.

\begin{abstract}
In Ecuador, there are montane forests of great biological importance that provide ecosystem services such as carbon storage. For this reason, we did this research for generate allometric models through by quantifying carbon in lower montane forests. We established one permanent plot of one hectare and measured all the trees with diameter at breast height $\left(\mathrm{DBH}_{1,30 \mathrm{~m}}\right) \geq 5 \mathrm{~cm}$. The variables that were used to generate the models were: DBH and total height (th). The tree biomass was $322.37 \mathrm{t} \mathrm{ha}^{-1}$ and the estimated total carbon was $161.07 \mathrm{t} \mathrm{ha}^{-1}$. In the allometric models of the arboreal stratum, the variables that were adjusted best were: $\mathrm{DBH}, \mathrm{DBH}^{2}$ and th. The botanical family with more biomass $\left(69.95 \mathrm{tha}^{-1}\right)$ and carbon $\left(34.98 \mathrm{t} \mathrm{C} \mathrm{ha}^{-1}\right)$ was Moraceae, which together with Lauraceae, Rubiaceae and Myristicaceae stored the 57\% carbon (91.55 t $\mathrm{C} \mathrm{ha}^{-1}$ ) in the lower montane forest. Of the 179 botanical species registered, Pseudolmedia laevigata, Nectandra reticulata, Otoba parvifolia, Vochysia paraensis, Ficus sp and Elaeagia obovata stored 51\% (81 t C ha ${ }^{-1}$ ) of carbon total estimated.
\end{abstract}

Keywords: biomass; ecosystem service; Lauraceae.

1 Ingeniera Forestal, MSc., Doctoranda del Programa de Pos-graduación en Ciencia Forestal, Universidade Estadual Paulista "Júlio de Mesquita Filho", Campus Botucatu, CEP 18610-385, Botucatu (SP), Brasil. caroladeicylo@, hotmail.com

2 Ingeniero Forestal, MSc., Profesor de la Carrera de Ingeniería Forestal, Universidad Nacional de Loja, Av. Pío Jaramillo Alvarado y Reinaldo Espinosa, Barrio La Argelia, Código Postal EC110110, Loja, Ecuador. byronph79@, yahoo.es

3 Ingeniero Forestal, Dr., Profesor de la Carrera de Ingeniería Forestal, Director del Herbario "Reinaldo Espinosa", Universidad Nacional de Loja, Av. Pío Jaramillo Alvarado y Reinaldo Espinosa, Barrio La Argelia, Código Postal EC110110, Loja, Ecuador. zhofre.aguirre@unl.edu.ec 


\section{INTRODUCCIÓN}

Los bosques son ecosistemas que capturan y conservan el carbono mediante la fijación y almacenamiento a través de la fotosíntesis (RÜGNITZ; CHACÓN; PORRO, 2009). La cantidad de carbono encima y abajo del suelo varía en función de variables bioclimáticas, de la composición florística, la edad y la densidad de población de cada estrato por comunidad vegetal, tasa de crecimiento, longevidad, ubicación, clima y rotación (ZAMBRANO; FRANQUIS; INFANTE, 2004; FONSECA; ALICE; REY, 2009; VALDÉS RAMÍREZ, 2012; RODRÍGUEZ; CARGUA, 2013; ARÉVALO, 2015).

La cuantificación de la biomasa en base al crecimiento de la vegetación en los ecosistemas forestales es importante para estimar el carbono almacenado y las tasas anuales de fijación, un tema relevante por sus implicaciones directas con el calentamiento global (CIFUENTES JARA, 2010; VÁSQUEZ; ARELLANO, 2012). Se ha demostrado que los ecosistemas forestales, con manejo adecuado, pueden secuestrar más carbono que otros ecosistemas terrestres, contribuyendo a la mitigación del cambio climático mediante la reducción de la tasa de aumento de $\mathrm{CO}_{2}$ en la atmósfera y estabilización de las concentraciones atmosféricas (BENNACEUR et al., 2005; YEPES QUINTERO et al., 2011).

Para estimar la cantidad de carbono en la biomasa de un bosque existen métodos directos e indirectos (BROW, 1997; BROW; CABARLE; LIVERNASH, 1997; SCHLEGEL; GAYOSO; GUERRA, 2001). Entre los métodos indirectos están las ecuaciones o modelos alométricos basados en análisis de regresión lineal o no lineal. Estos utilizan variables medidas en el campo denominadas regresoras o independientes tales como: diámetro a la altura del pecho (DAP), altura comercial (hc) y altura total (ht), incremento diamétrico, área basal y densidad específica de la madera (CHAVE et al., 2005; JADÁN; TORRES; GÜNTER, 2012).

En el Ecuador, según el Ministerio del Ambiente (MAE) (ECUADOR, 2012a), existen bosques de tierras bajas de gran importancia biológica con una cobertura vegetal significativa para la conservación y provisión de servicios ecosistémicos como el almacenamiento de carbono, el cual, tendría muchas implicaciones negativas en el momento de ser liberado a la atmósfera por actividades antrópicas. Uno de estos ecosistemas es el bosque montano bajo que forma parte de la cuenca del río Amazonas (un verdadero sumidero de carbono). Pese a su importancia, este ecosistema forestal se encuentra amenazado, debido a las altas tasas de deforestación, degradación y el avance de la frontera agrícola (LAMBIN; GEIST; LEPERS, 2003; MALHI; PHILLIPS, 2004; ORGANIZACIÓN DE LAS NACIONES UNIDAS PARA LA AGRICULTURA Y LA ALIMENTACIÓN, 2007b; BACCINI et al., 2012). Por ejemplo, la deforestación promedio en Ecuador para el período 2000 - 2008 fue de 77.647 ha año ${ }^{-1}$ mientras que para el período 2008 - 2012 fue de 65.880 ha año ${ }^{-1}$ (ECUADOR, 2014), específicamente en los bosques amazónicos la deforestación promedio para el periodo de 2000 - 2008 fue de 16.430 ha año $^{-1}$ (ECUADOR, 2012b). Por lo tanto la caracterización y valoración de sus servicios ecosistémicos, como el carbono almacenado, es un tema relevante para la política nacional de cambio climático (ECUADOR, 2012a), así como, para disminuir las tasas de deforestación de estos ecosistemas. Los objetivos de esta investigación fueron: cuantificar la biomasa almacenada encima del suelo, ajustar modelos alométricos para estimar la biomasa y determinar las familias y especies botánicas que acumulan la mayor cantidad de carbono, en el bosque montano bajo del Sur de Ecuador.

\section{MATERIALES Y MÉTODOS}

\section{Ubicación del área de estudio}

El estudio se realizó en el bosque montano bajo de la microcuenca "El Padmi" de la Universidad Nacional de Loja, ubicado en el corredor fluvial del Río Zamora, entre 775 - $1150 \mathrm{~m}$ de altitud, con precipitación media anual de $1978 \mathrm{~mm}$ año ${ }^{-1}$, clima semicálido húmedo, temperatura media anual de $23{ }^{\circ} \mathrm{C}$ y suelos jóvenes del grupo entisoles e inceptisoles, en la clasificación de la FAO este orden de suelos entra típicamente en el Grupo de Cambisoles (SIERRA et al., 1999; CERÓN et al., 2003; VALAREZO, 2004; ORGANIZACIÓN DE LAS NACIONES UNIDAS PARA LA AGRICULTURA Y LA ALIMENTACIÓN, 
2007a). Según Holdridge (1967), el área de estudio pertenece a la zona de vida bosque muy húmedo tropical (Figura 1).

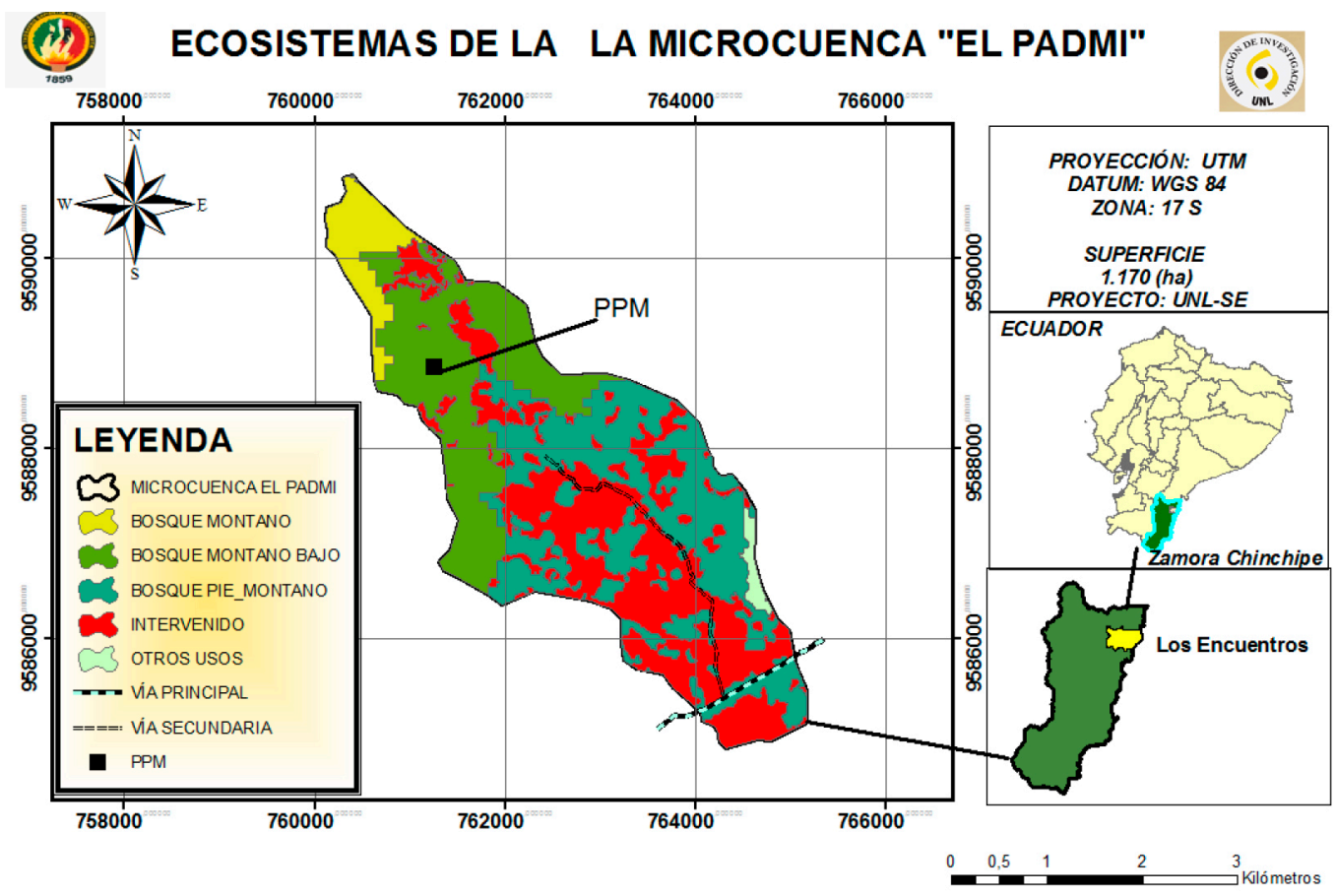

FIGURA 1: Ubicación geográfica del área de estudio, microcuenca "El Padmi, Zamora Chinchipe, sur de Ecuador.

FIGURE 1: Geographical location of the study area of the watershed "The Padmi, Zamora Chinchipe, southernEcuador.

\section{Registro y análisis de datos}

Se instaló una parcela permanente considerando la topografía del terreno y la heterogeneidad del bosque. La parcela permanente de 1 ha fue subdivida en 25 unidades de muestreo de $400 \mathrm{~m}^{2}$, donde se inventarió los individuos $\geq 5 \mathrm{~cm}$ de DAP, para obtener el volumen total.

Para estimar el carbono almacenado en la biomasa de los árboles, palmas y helechos arborescentes del bosque se aplicó la metodología propuesta por Ravindranath y Ostwald (2008), quienes indican que la biomasa se obtiene del volumen de cada árbol multiplicándolo por la densidad de la madera (ecuación 1), esta última variable fue obtenida de lista de densidades de maderas de la FAO (ZANNE et al., 2009). Para obtener el carbono fijado por árbol (ecuación 2) la biomasa se multiplica por el factor de conversión 0,5 propuesto por el Grupo Intergubernamental de Expertos sobre el Cambio Climático (IPCC) (PENMAN et al., 2003). Los valores obtenidos fueron expresados en toneladas de carbono por hectárea ( $\left.\mathrm{t} \mathrm{C} \mathrm{ha}^{-1}\right)$. El carbono total almacenado se calculó sumando el carbono en cada uno de los compartimentos de almacenamiento (biomasa arbórea, palmas y helechos arborescentes).

$$
\begin{aligned}
& B=V . \rho \\
& C_{\text {fijado }}=B . f
\end{aligned}
$$

Donde: $\mathrm{B}=$ biomasa en $\mathrm{tha}^{-1} ; \mathrm{V}=$ volumen del árbol en $\mathrm{m}^{3} ; \rho=$ densidad de la madera en $\mathrm{m}^{3} \mathrm{~kg}^{-1} ; \mathrm{C}_{\text {fijado }}$ $=$ carbono fijado en $\mathrm{t} \mathrm{Ch} \mathrm{h}^{-1} ; f=$ factor de conversión de biomasa a carbono 0,5 (IPCC, 2006).

Para determinar las variables dendrométricas que influyen sobre la presencia y magnitud de la biomasa se calculó la correlación de Pearson $(\mathrm{p}<0,05)$ entre diámetro a la altura del pecho $\left(\mathrm{DAP}_{1.30 \mathrm{~m}}\right)$, altura total $(\mathrm{ht})$, área basal (G) y volumen total (Vt). 


\section{Ajuste y selección de los modelos}

Para construir los modelos se utilizó el programa estadístico Infostat profesional versión 2013 (DI RIENZO et al., 2011). La selección de las ecuaciones alométricas mejor ajustadas se realizó aplicando la metodología propuesta por Segura y Andrade (2008) y Fonseca, Alice y Rey (2009) que proponen los siguientes pasos: los datos que se usen en el análisis de regresión deben cumplir los supuestos de normalidad, independencia y homogeneidad de varianzas. En caso de no cumplirse algunos de ellos, entonces se transforman las variables usando logaritmos (naturales y en base 10), potencias y raíces. Walpole y Myers (1992) indican que una gráfica de valores observados en función de la variable independiente puede indicar la necesidad de transformar los datos a logaritmos. Cuando no sea posible linearizar mediante transformaciones.

Para seleccionar el modelo se consideraron las siguientes variables:

- Cálculo de indicadores de ajuste: coeficiente de determinación ajustado $\left(\mathrm{R}^{2}\right)$. Aquellos modelos que presentaron, al menos, un coeficiente no significativo $(\mathrm{p}>0,05)$ respecto a una variable utilizada como independiente o regresora fueron eliminados.

- Cálculo de indicadores de capacidad predictiva de los modelos: Error Cuadrático Medio Ponderado (ECMP), Criterio de Información Akaike (AIC) y Criterio de información Bayesiano (BIC).

- Se calificó cada modelo en base a la escala de calificación propuesta por Segura y Andrade (2008). El modelo con menor puntaje en base a la sumatoria de los indicadores fue seleccionado como el mejor, en base a lo siguiente: en el caso del $\mathrm{R}^{2}$ será el de mayor valor y tomará el valor 1, cuando se aproxime a este (escala de 0-1) y en los indicadores de predicción (ECMP; AIC y BIC) los que más se acercan a cero. Los valores de 2, 3, 4 etc., corresponderán al valor del indicador que se aleja de uno $\left(\mathrm{R}^{2}\right)$ o del cero.

Finalmente, se determinó la familia y especie botánica que almacenan la mayor cantidad de carbono en el bosque montano bajo, para lo cual se realizó un análisis estadístico multivariado (ANAVA) utilizando el programa estadístico Infostat profesional versión 2013 (DI RIENZO et al., 2011).

\section{RESULTADOS Y DISCUSIÓN}

\section{Biomasa encima del suelo}

La biomasa y carbono arbóreo dependen del área basal, altura total y número de individuos de los bosques, como se evidencia en el carbono estimado en el bosque montano bajo, el cual fue de 161,07 t C ha $^{-1}$ (Tabla 1). Los resultados hallados se encuentran en el rango que señala el EcoSecurities (2007), para los bosques tropicales montanos que están entre 100 y $200 \mathrm{tC} \mathrm{ha}^{-1}$. Así mismo, estos resultados son corroborados por Unger, Homeier y Leuschner (2012) quienes determinaron $344 \pm 17 \mathrm{t} \mathrm{ha}^{-1}$ de biomasa respecto al área basal, al transformar la biomasa estimada por Unger, Homeier y Leuschner (2012) a carbono mediante el factor de conversión de 0,5 propuesto por el IPCC tenemos un valor equivalente a $172 \pm 8,5 \mathrm{t} \mathrm{C} \mathrm{ha-1}$.

Por otro lado, Honorio-Coronado, Vega-Arenas y Corrales-Medina (2015), calcularon un promedio de 193,9 $\pm 55,6 \mathrm{t} \mathrm{ha}^{-1}$ de biomasa en bosques aluviales de la amazonia peruana, valor diferente al reportado en este estudio para el bosque montano bajo de Ecuador, a pesar de ser ecosistemas con características climáticas similares. En cambio, en estudios realizados en bosques de la amazonia de Brasil, Castilho et al. (2006), determinaron en promedio $327,8 \mathrm{t} \mathrm{ha}^{-1}$ de biomasa y Nogueira (2006) estimo 320,8 $\mathrm{t} \mathrm{ha}^{-1} \mathrm{de}$ biomasa, valores que concuerdan con los resultados de esta investigación. También, Bertzky et al. (2010) en su estudio sobre carbono, biodiversidad y servicios ecosistémicos realizado en Ecuador, señala que los bosques siempreverdes de tierras bajas de la amazonia almacena mayor cantidad de carbono en relación a ecosistemas como los páramos, lo cual se contrasta con los datos encontrados en la presente investigación.

Sin embargo, Hofstede (1999) menciona que la biomasa de la selva tropical almacena $250 \mathrm{t} \mathrm{C} \mathrm{ha}^{-1}$ valor distante al reportado en esta investigación. También, los resultados de esta investigación (bosque montano bajo) difieren con los encontrados por Arévalo (2015), en su investigación realizada en el estrato arbóreo del bosque natural Tinajillas - Limón Indanza en Ecuador, quien estimo 41 t C ha ${ }^{-1}$. Así mismo, los 
resultados obtenidos en el bosque montano bajo son diferentes a los reportados por Ponce et al. (2009), en su estudio realizado en la cordillera del Cóndor en Ecuador, cuya estimación de biomasa y carbono fue de 43,7 tha- y 21,8 t C ha ${ }^{-1}$, respectivamente.

La diferencia en los resultados posiblemente se debe al número de individuos evaluados, que difieren notablemente entre los estudios realizados en la cordillera del Cóndor, bosque natural Tinajillas y el bosque montano bajo. En el bosque montano bajo se evaluaron 1.408 individuos ha ${ }^{-1}$ y 52 familias botánicas para la cuantificación del almacenamiento de carbono (Tabla 1), en cambio, en el estudio de la cordillera del Cóndor se evaluaron 512,9 individuos ha ${ }^{-1}$ y 37 familias botánicas, y en el bosque natural Tinajillas - Limón Indanza se evaluaron 925 individuos y 22 familias botánicas.

TABLA 1: Biomasa total encima del suelo y reserva de carbono de la vegetación en el bosque montano bajo del sur de Ecuador.

TABLE 1: Total aboveground biomass and carbon stock of vegetation in low montane forest in southern Ecuador.

\begin{tabular}{ccc}
\hline VeGetación & Biomasa t ha $^{-1}$ & Carbono $\mathrm{t} \mathrm{C} \mathrm{ha}^{-1}$ \\
\hline Árboles & 318,63 & 159,31 \\
Palmas & 3,29 & 1,64 \\
Helechos & 0,25 & 0,12 \\
\hline arborescentes & 322,17 & 161,07 \\
\hline Total & &
\end{tabular}

Cuando analizamos las correlaciones (Pearson, $\mathrm{p}<0,05$ ) entre la biomasa y las variables dasométricas de los árboles constatamos que los resultados estaban fuertemente correlacionados (Tabela 2). Las variables: DAP $\left(\mathrm{m} \mathrm{ha}^{-1}\right)$, área basal $\left(\mathrm{m}^{2} \mathrm{ha}^{-1}\right)$ y volumen $\left(\mathrm{m}^{3} \mathrm{ha}^{-1}\right)$ explican significativamente $(\mathrm{p}<0,05)$ la cantidad de carbono encima del suelo debido a que están fuertemente correlacionadas con la biomasa. En cambio, la altura $(\mathrm{m})$ presenta relación media cuya correlación es moderada con la biomasa del bosque montano bajo (Tabla 2). Según, Pearson et al. (2007), las variables de rendimiento dasométricas están altamente relacionadas con la biomasa de un ecosistema, lo que es ratificado con los resultados encontrados en el bosque montano bajo. En cambio, Lal (2005), indica que la biomasa y el almacenamiento del carbono están influenciados por complejas interacciones de clima, suelo, manejo y especies vegetales.

TABLA 2: Valores del coeficiente de correlación de Pearson (R) entre la biomasa y las variables de rendimiento para el bosque montano bajo, sur de Ecuador.

TABLE 2: Values of the Pearson correlation coefficient (R) between biomass and performance variables for the lower montane forests, southern Ecuador.

\begin{tabular}{ccc}
\hline VARIABLE A & Variable B & $\mathrm{R}^{*}$ \\
\hline Biomasa $\left(\mathrm{t} \mathrm{ha}^{-1}\right)$ & DAP $(\mathrm{m})$ & 0,79 \\
Biomasa $\left(\mathrm{t} \mathrm{ha}^{-1}\right)$ & Altura $(\mathrm{m})$ & 0,54 \\
Biomasa $\left(\mathrm{t} \mathrm{ha}^{-1}\right)$ & Área basa $\left(\mathrm{m}^{2} \mathrm{ha}^{-1}\right)$ & 0,90 \\
Biomasa $\left(\mathrm{t} \mathrm{ha}^{-1}\right)$ & Volumen $\left(\mathrm{m}^{3} \mathrm{ha}^{-1}\right)$ & 0,97 \\
\hline
\end{tabular}

*Valores de $\mathrm{R}$ superiores a 0,70 (positivo o negativo) indica que las variables están fuertemente correlacionadas, de 0,30 a 0,70 (positivo o negativo) indica que la correlación es moderada y de 0 a 0,30 se considera como débil correlación (RÜGNITZ et al., 2009). 


\section{Modelos alométricos para estimar biomasa encima del suelo}

Los modelos alométricos generados para estimar la biomasa aérea en el bosque montano bajo de la microcuenca El Padmi se indican en la Tabla 3, todos los modelos seleccionados indican un $\mathrm{R}^{2}$ ajustado superior al $80 \%$, los parámetros de cada modelo fueron significativos $(\mathrm{p}<0,0001)$.

TABLA 3: Modelos alométricos ajustados para el estrato arbóreo del bosque montano bajo del Sur de Ecuador. TABLE 3: Allometric models adjusted to the tree layer of the lower montane forests of Southern Ecuador.

\begin{tabular}{lllcccc}
\hline N. & \multicolumn{1}{c}{ Modelo } & $\mathrm{R}^{2}$ & $\mathrm{R}^{2} \mathrm{Aj}$ & $\mathrm{ECMP}(\%)$ & $\mathrm{AIC}$ & $\mathrm{BIC}$ \\
\hline 1 & $\mathrm{~B}=0,00062 * \mathrm{DAP}^{2}-0,07$ & 0,80 & 0,80 & 0,17 & 1370,23 & 1385,98 \\
2 & $\mathrm{~B}=0,00088(\mathrm{DAP})^{2}-0,03$ (DAP) $+0,04(\mathrm{ht})-0,08$ & 0,84 & 0,84 & 0,14 & 938,39 & 964,64 \\
3 & $\mathrm{Ln}(\mathrm{B})=1,00 \mathrm{Ln}(\mathrm{DAP})+1,95 \mathrm{Ln}(\mathrm{ht})-10,37$ & 0,98 & 0,98 & 0,07 & 178,91 & 199,91 \\
\hline
\end{tabular}

Donde: $\mathrm{N}^{\mathrm{o}}=$ número de modelo alométrico generado; $\mathrm{B}=$ biomasa arbórea $\left(\mathrm{t} \mathrm{ha} \mathrm{a}^{-1}\right) ; \mathrm{N}=1408$ individuos, $\mathrm{DAP}=$ diámetro a la altura del pecho $(\mathrm{m}) ; \mathrm{ht}=$ altura total $(\mathrm{m}) ; \mathrm{G}=$ área basal $\left(\mathrm{m}^{2}\right) ; \mathrm{V}=$ volumen $\left(\mathrm{m}^{3}\right) ; \mathrm{R}^{2}=$ coeficiente de determinación, $\mathrm{R}^{2} \mathrm{Aj}=$ coeficiente de determinación ajustado; $\mathrm{ECMP}(\%)=$ Error cuadrático medio ponderado; $\mathrm{AIC}=$ Criterio de información Akaike; $\mathrm{BIC}=$ Criterio de información Bayesiano.

En la Figura 2 se muestra la distribución gráfica del primer modelo generado y ajustado según se indica en la Tabla 3. Se realizó la representación gráfica del primer modelo generado debido a que tiene un buen coeficiente de determinación ajustado $\left(\mathrm{R}^{2} \mathrm{Aj}\right)$ y además utiliza una sola variable explicativa, el DAP, la cual es una de las variables dasométricas medidas en el campo con un alto valor de confiabilidad, como menciona Chave et al. (2005), el diámetro es la variable más importante en los modelos alométricos para la estimación de carbono. También, Nogueira et al. (2008) indican que utilizar el diámetro en modelos alométricos es una ventaja importante, para el uso práctico de la ecuación en la estimación de biomasa seca encima del suelo.

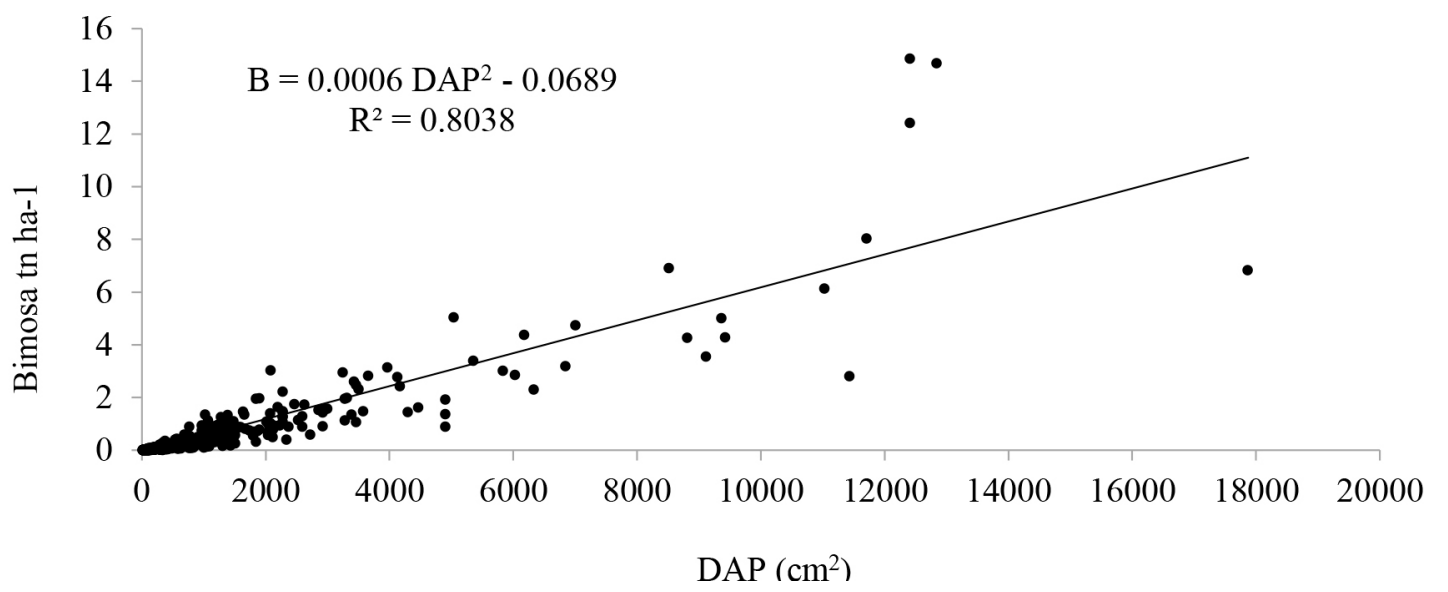

FIGURA 2: Relación entre la biomasa estimada y el diámetro a altura del pecho ( $\left.\mathrm{DAP}^{2}\right)$, en arboles $\geq 5 \mathrm{~cm}$ de DAP del bosque montano bajo del Sur de Ecuador.

FIGURE 2: Relationship between estimated biomass and diameter at breast height $\left(\mathrm{DBH}^{2}\right)$, in trees $\geq 5 \mathrm{~cm}$ of DAP of low montane forest in southern Ecuador. 
Al comparar las tendencias de la acumulación de biomasa determinados en este estudio, con modelos generados para bosque montano bajo de otros lugares, como el modelo ajustado por Chave et al. (2005) y el modelo generado por Álvarez et al. (2012), se observó semejanzas en las tendencias de acumulación de biomasa en estos ecosistemas (Figura 3), esto indica que los modelos generados en esta investigación son factibles de aplicar como una herramienta para cuantificación de biomasa en ecosistemas tropicales. Además, según Montero y Montagnini (2005), el uso de ecuaciones alométricas permite el cálculo de la biomasa de una especie forestal de manera no destructiva y extrapolable a situaciones de crecimiento similares a las reales.

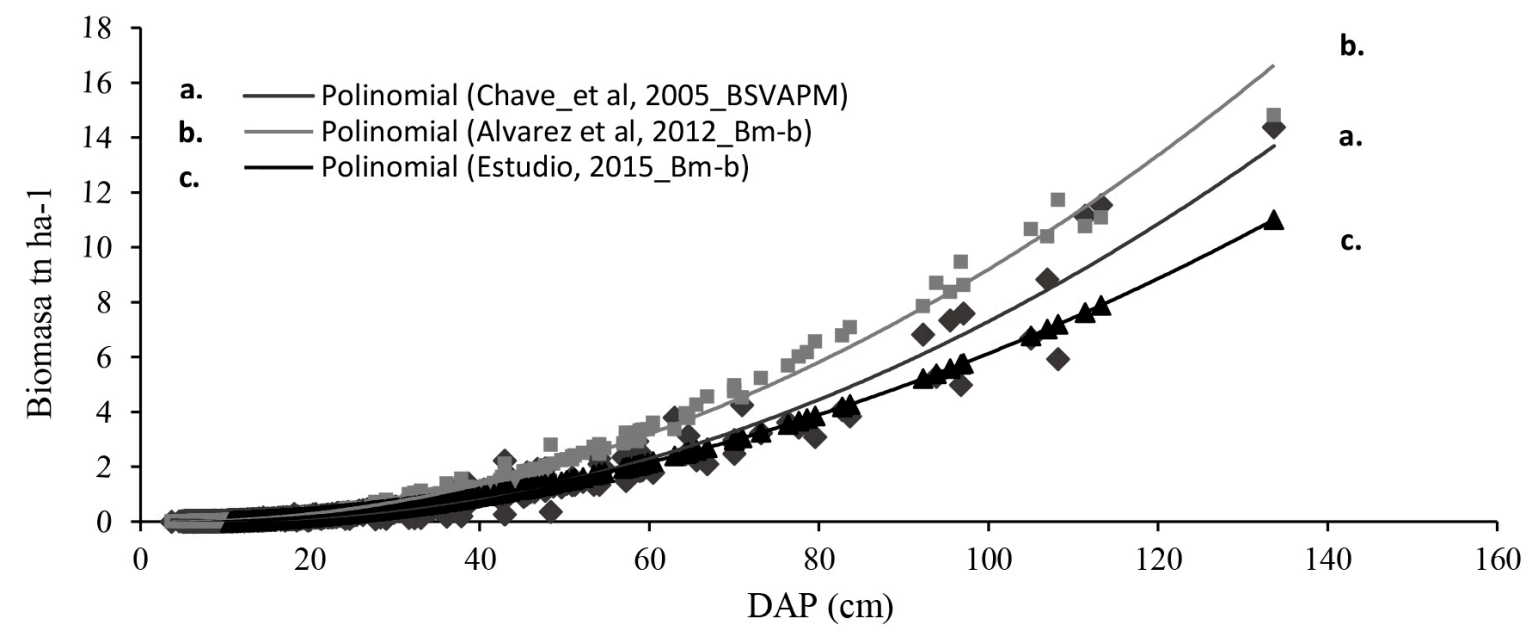

FIGURA 3: Representaciones de dispersión de la variable biomasa en bosques montanos bajos con: a) modelo alométrico generado por Chave et al. (2005); b) modelo alométrico generado por Álvarez et al. (2012); y c) modelo alométrico generado en este estudio.

FIGURE 3: Representations of dispersion of the variable biomass in the lower montane forests: a) allometric model generated by Chave et al. (2005); b) allometric model generated by Álvarez et al. (2012); and c) allometric model generated in this study.

\section{Almacenamiento de carbono en la composición florística}

En el bosque montano bajo "El Padmi" existe diferencia estadística $(\mathrm{p}<0,05)$ entre dos grupos de familias botánicas según el almacenamiento de carbono en su biomasa, las variables que posiblemente expliquen esta división son: densidad de la madera, número de individuos por especies y el DAP, lo cual es corroborado por Fonseca, Alice y Rey (2009). En la Figura 4, se indica la media de almacenamiento de biomasa según los dos grupos taxonómicos diferenciados, el grupo uno en promedio almacena $0,05 \mathrm{t} \mathrm{ha}^{-1}$ y el grupo dos $0,28 \mathrm{tha}^{-1}$. 


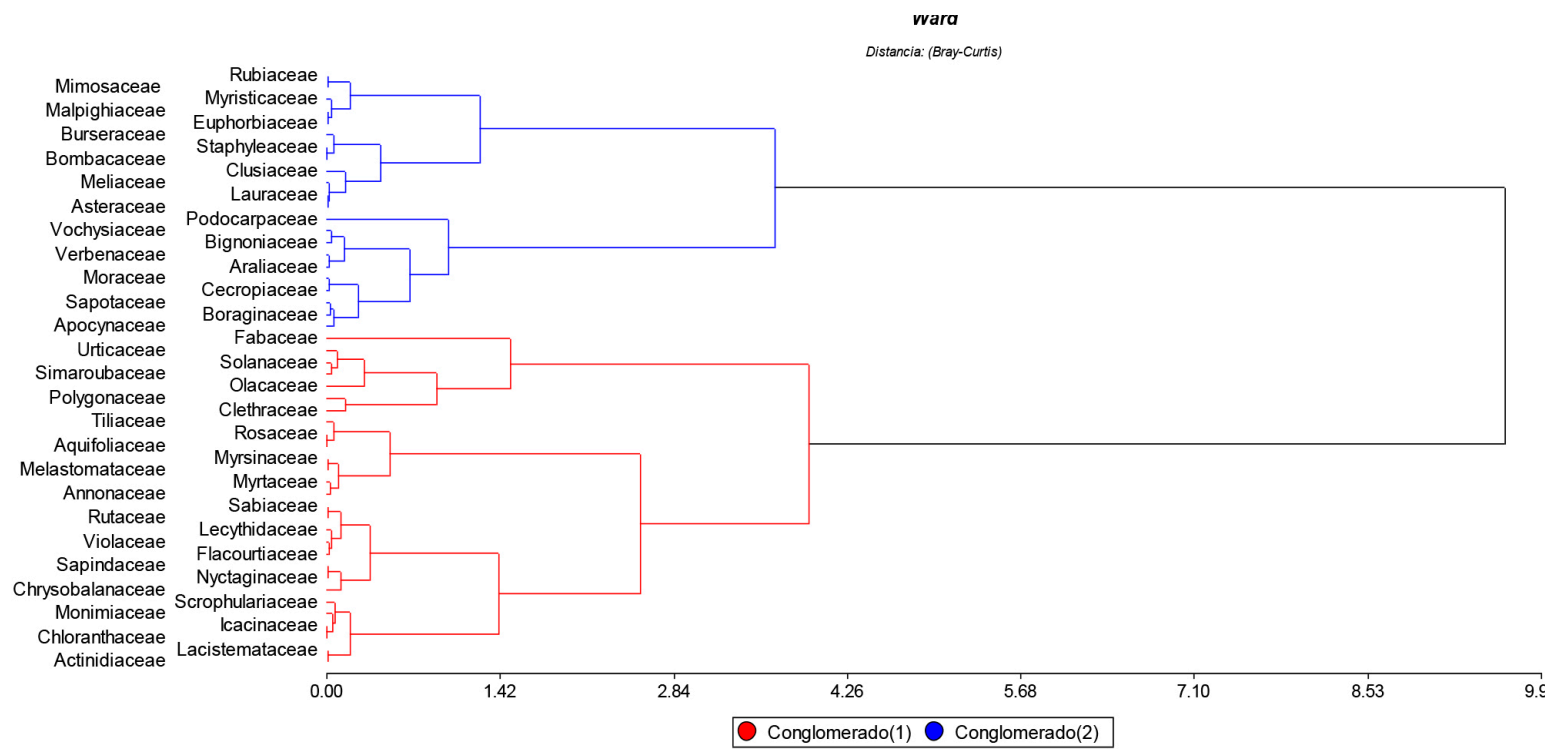

FIGURA 4: Dendrograma de las familias con mayor cantidad de carbono acumulado aplicando el método de Ward y distancia de Bray-Curtis.

FIGURE 4: Dendrogram of families with a higher amount of accumulated carbon using the method of Ward and Bray-Curtis distance.

La familia que almacena la mayor cantidad de biomasa $\left(74,83 \mathrm{t} \mathrm{ha}^{-1}\right)$ y carbono $\left(37,42 \mathrm{t} \mathrm{C} \mathrm{ha}^{-1}\right)$ es Moraceae. Las familias Moraceae, Lauraceae, Myristicaceae y Rubiaceae acumulan el $57 \%$ de carbono total $\left(91,55 \mathrm{t} \mathrm{C} \mathrm{ha}^{-1}\right)$ del bosque montano bajo (Figura 5). Estos resultados son similares a los determinados en la amazonia peruana por Ureta (2015). Al referirnos a las especies botánicas, Pseudolmedia laevigata almacena la mayor cantidad de biomasa $\left(55,23 \mathrm{t} \mathrm{ha}^{-1}\right)$ y carbono $\left(27,61 \mathrm{t} \mathrm{C} \mathrm{ha}^{-1}\right)$ de las 179 especies evaluadas. Esta especie, en conjunto con Nectandra reticulata, Otoba parvifolia, Vochysia paraensis, Ficus sp y Elaeagia obovata almacenan el 51\% del carbono ( $\left.81 \mathrm{t} \mathrm{C} \mathrm{ha-}^{-1}\right)$ del bosque montano bajo (Figura 6). Los resultados encontrados demuestran que los bosques amazónicos almacenan elevadas cantidades de biomasa y carbono en su composición florística y estructura, sin embargo, esto depende de la fisiografía, tal y como lo atribuye Phillips et al. (1998) y Ureta (2015).

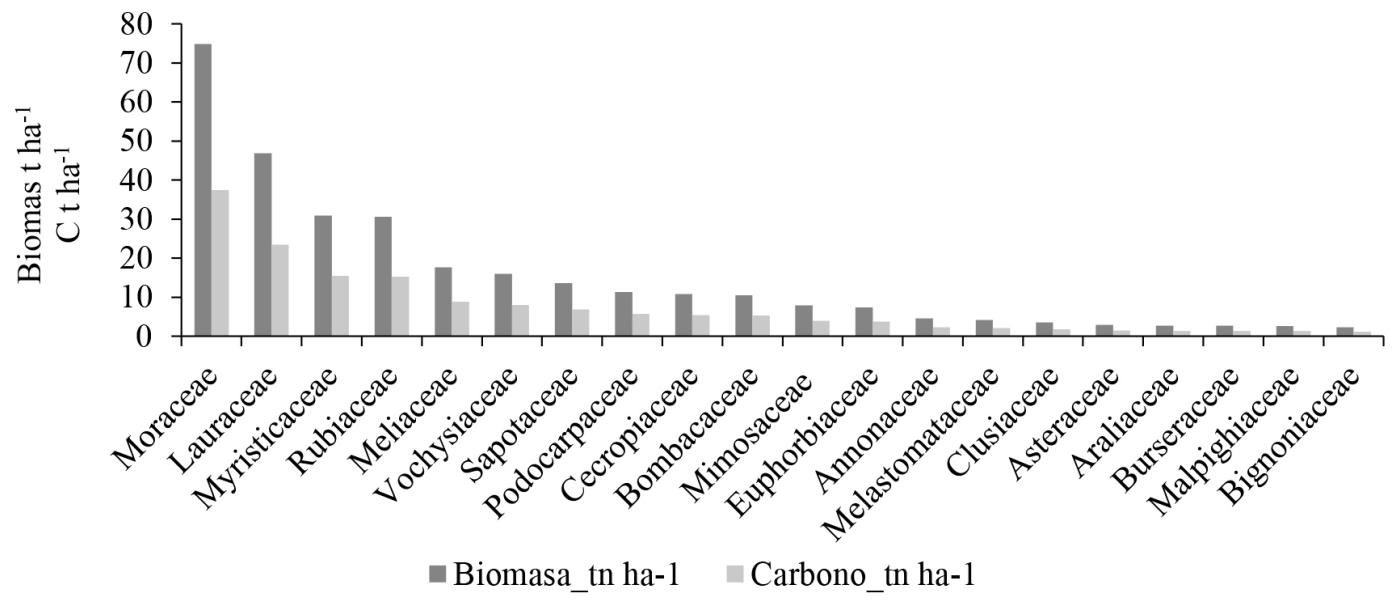

FIGURA 5: Familias con la mayor cantidad de carbono en el bosque montano bajo.

FIGURE 5: Families with the highest amount of carbon in the lower montane forests. 


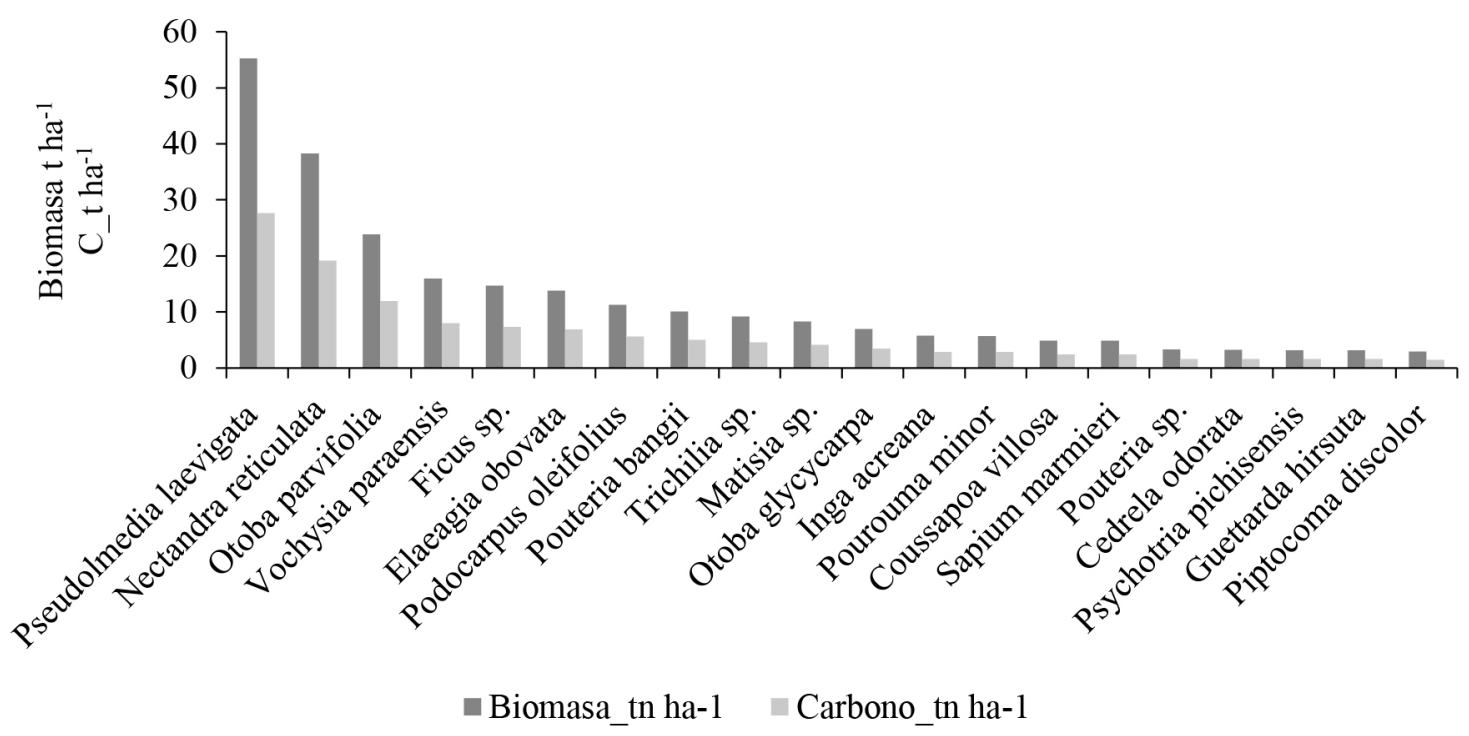

FIGURA 6: Especies botánicas con mayor cantidad de carbono en el bosque montano bajo.

FIGURE 6: Botanical species with more carbon in the lower montane forests.

\section{CONCLUSIONES}

El bosque montano bajo almacena $161,07 \mathrm{t} \mathrm{C}$ ha-1 en 179 especies arbóreas y 52 familias.

Los modelos alométricos ajustados para predecir la biomasa en el estrato arbóreo del bosque montano bajo mostraron coeficientes de determinación (R ajustados) mayores al $80 \%$. Las variables regresoras que mejor se ajustaron en la ecuación para la predicción de la biomasa aérea total fueron el diámetro a la altura de pecho al cuadrado $\left(\mathrm{DAP}^{2}\right)$ y altura total (ht) tanto en escala lineal como logarítmica.

En el bosque montano bajo evaluado en el sur del Ecuador existen dos grupos de familias significativamente diferentes $(\mathrm{p}<0,05)$ de acuerdo al almacenamiento de carbono; siendo la familia Moraceae $\left(74,83 \mathrm{t} \mathrm{ha}^{-1} ; 37,42 \mathrm{t} \mathrm{C} \mathrm{ha}^{-1}\right)$ y la especie Pseudolmedia laevigata Trécul $\left(55,23 \mathrm{t} \mathrm{ha}^{-1} ; 27,61 \mathrm{t} \mathrm{C}\right.$ ha $^{-1}$ ) las que almacenan la mayor cantidad de biomasa y carbono respectivamente.

\section{AGRADECIMIENTOS}

Los autores agradecen a la Dirección de Investigaciones de la Universidad Nacional de Loja por el financiamiento del proyecto de investigación "Evaluación del potencial de los servicios ecosistémicos de la microcuenca El Padmi, Zamora Chinchipe - Ecuador", lo que permitió realizar este trabajo. A la comunidad "El Padmi" por la facilidad de los terrenos donde se instaló la parcela de estudio.

\section{REFERENCIAS}

ÁLVAREZ, E. et al. Tree above-ground biomass allometries for carbon stocks estimation in the natural forests of Colombia. Forest Ecology and Management, Amsterdam, v. 267, p. 297-308, 2012. ARÉVALO, C. Medición de carbono del estrato arbóreo en un área del bosque natural Tinajillas Limón Indanza. 2015. 187 f. Tesis (Ingeniería) - Universidad Politécnica Salesiana, Cuenca, 2015. BACCINI, A. et al. Estimated carbon dioxide emissions from tropical deforestation improved by carbon density maps. Journal of Nature Climate Change, London, v. 2, n 3, p. 182-185, 2012. 
BENNACEUR, K. et al. El hidrógeno: ¿Un futuro portador energético? Gas, Estados Unidos, v. 25, n 20.1, p. $34-47,2005$.

BERTZKY, M. et al. Carbon, biodiversity and ecosystem services: exploring co-benefits. Ecuador: UNEP; WCMC, 2010. $24 \mathrm{p}$.

BROWN, P.; CABARLE, B.; LIVERNASH, R. Carbon counts: estimating climate change mitigation in forestry projects. United States: World Resources Institute, 1997. 25 p.

BROWN, S. Los bosques y el cambio climático: el papel de los terrenos forestales como sumideros de carbono. In: CONGRESO MUNDIAL FORESTAL: RECURSOS FORESTALES Y ARBOLES, 11., 1997, Ankara. Acta... [s. 1.: s. n.], 1997. p. 13-22.

CERÓN, C. et al. Leñosas frecuentes en la Estación Experimental El Padmi de la Universidad Nacional de Loja. Loja: [s. n.], 2003. 40 p.

CIFUENTES JARA, M. ABC del cambio climático en Mesoamérica. Turrialba: CATIE, 2010. 71 p. (Serie Técnica, 383).

CHAVE, J. et al. Tree allometry and improved estimation of carbon stocks and balance in tropical forests. Journal Ecosystem Ecology, Switzerland, v. 145, n 1, p. 87-99, 2005.

CASTILHO, C. V. et al. Variation in aboveground tree live biomass in a central Amazonian Forest: effects of soil and topography. Forest Ecology and Management, Amsterdam, v. 234, p. 85-96, 2006.

DI RIENZO, J. et al. InfoStat versión 2011. Grupo InfoStat, FCA. Córdoba: Universidad Nacional de Córdoba, 2011. Disponible en: 〈http://www. infostat. com. ar〉. Acceso en: 15 oct. 2015.

ECOSECURITIES. Policy brief: REDD Policy Scenarios and Carbon Markets. Oxford: [s. n.], 2007. 9 p. ECUADOR. Ministerio del Ambiente. Estrategia Nacional de Cambio Climático del Ecuador 20122025. 2012a. Disponible en: 〈http://www.redisas.org/pdfs/ENCC.pdf〉. Acceso en: 18 set. 2015.

ECUADOR. Ministerio del Ambiente. Línea Base de Deforestación del Ecuador Continental. Quito: MAE, 2012b. 32 p.

ECUADOR. Ministerio del Ambiente. Plan Nacional de Restauración 2014-2017. Quito: MAE, 2014. $50 \mathrm{p}$.

FONSECA, W.; ALICE, F.; REY, J. M. Modelos para estimar la biomasa de especies nativas en plantaciones y bosques secundarios en la zona Caribe de Costa Rica. Revista Bosque, Valdivia, v. 30, n 1, p. 36-47, 2009.

HOFSTEDE, R. El páramo como espacio para la fijación de carbono atmosférico. En: EL PÁRAMO como espacio de mitigación de carbono atmosférico. Quito: GTP; Abya-Yala, 1999. 57 p. (Serie Páramo, 1).

HOLDRIDGE, L. R. Life zone ecology. San José: Tropical Science Center, 1964. 206 p.

HONORIO-CORONADO, E. N.; VEGA-ARENAS, J. E.; CORRALES-MEDINA, M. N. Diversidad, estructura y carbono de los bosques aluviales del noreste peruano. Folia Amazónica, Perú, v. 24, n. 1, p. 55-70, 2015.

LAL, R. Forest soils and carbon sequestration. Forest Ecology and Management, Amsterdam, v. 220, p. 242-258, 2005.

JADÁN, O.; TORRES, B.; GÜNTER, S. Influencia del uso de la tierra sobre almacenamiento de carbono en sistemas productivos y bosque primario en Napo, Reserva de Biósfera Sumaco, Ecuador. Revista Amazónica: Ciencia y Tecnología, Ecuador, v. 1, n. 3, p. 173-186, 2012.

LAMBIN, E. F.; GEIST, H. J.; LEPERS, E. Dynamics of land - use and land - cover change in tropical regions. Annual Review of Environment and Resources, United States of America, v. 28, n 1, p. 205-241, 2003.

MALHI, Y.; PHILLIPS, O. L. Tropical forests and global atmospheric change: a synthesis. Journal Philosophical Transactions of the Royal Society of London. Series B: Biological Sciences, London, v. 359, n 1443, p. 549-555, 2004.

MONTERO, M.; MONTAGNINI, F. Modelos alométricos para la estimación de biomasa de diez especies 
nativas en plantaciones en la región Atlántica de Costa Rica. Revista Recursos Naturales y Ambiente, Costa Rica, n. 45, p. 112-119, 2005.

NOGUEIRA, A. Variação da densidade, área basal e biomassa de lianas em $64 \mathbf{k m}^{2}$ de floresta de terra-firme na Amazônia central. 2006. 55 f. Dissertação (Mestrado) - Instituto Nacional de Pesquisas da Amazonia; Fundação Universidade do Amazonas, Manaus, 2006.

NOGUEIRA, E. M. et al. Estimates of forest biomass in the Brazilian Amazon: new allometric equations and adjustments to biomass from wood-volume inventories. Forest Ecology and Management, Amsterdam, v. 256, p. 1853-1867, 2008.

ORGANIZACIÓN DE LAS NACIONES UNIDAS PARA LA AGRICULTURA Y LA ALIMENTACIÓN. Base referencial mundial del recurso suelo. 2007a. (Informes sobre Recursos Mundiales de Suelos, $\mathrm{n}$. 103). Disponible: <http://www.fao.org/3/a-a0510s.pdf >. Acceso: 11 abr. 2017

ORGANIZACIÓN DE LAS NACIONES UNIDAS PARA LA AGRICULTURA Y LAALIMENTACIÓN. Los bosques y el cambio climático. 2007b. (Departamento forestal Boletín Informativo). Disponible en: 〈www.fao.org/forestry/climatechange/es.infofaospanishlosbosquesyelcambioclimatico.pdf〉. Acceso en: 15 set. 2015.

PEARSON, T. R. H.; BROWN, S. L.; BIRDSEY, R. A. Measurement guidelines for the sequestration of forest carbon. [s. 1.]: USDA Forest Service's Northern Global Change Research Program, 2007. 47 p. Disponible en: 〈http://www.nrs.fs.fed.us/pubs/gtr/gtr_nrs18.pdf〉. 2007. Acceso en: 19 dic. 2015.

PENMAN, J. et al. Good practice guidance for land use, land-use change and forestry. [s. 1.]: Institute for Global Environmental Strategies, 2003. 590 p.

PHILLIPS, O. L. et al. Changes in the carbon balance of tropical forests: evidence from long-term plots. Science, United States of America, v. 282, n. 5388, p. 439-442, 1998.

PONCE, E. et al. Inventario forestal y de materia orgánica como línea base para la estimación de la cantidad de carbono fijado en la cordillera del Cóndor. [s. 1.]: Fundación Natura, 2009. 67 p.

RAVINDRANATH, N. H.; OSWALD, M. Carbon inventory methods: handbook for greenhouse gas inventory, carbon mitigation and roudwood production projects. [s. 1.]: Springer Science and Business media, 2008. $306 \mathrm{p}$.

RODRÍGUEZ, C.; CARGUA, F. Elaboración de un inventario forestal multipropósito con énfasis en el contenido de carbono de las diferentes clases de uso de tierra, parroquia Achupallas, cantón Alausí, provincia de Chimborazo. 2013. 199 f. Tesis (Ingeniería) - Escuela Superior Politécnica de Chimborazo, Riobamba, 2013.

RÜGNITZ, M. T.; CHACÓN, M. L.; PORRO, R. Guía para la determinación de carbono en pequeñas propiedades rurales. 1. ed. Lima: Centro Mundial Agroflorestal; Consórcio Iniciativa Amazônica, 2009. $79 \mathrm{p}$.

SCHLEGEL, B.; GAYOSO, J.; GUERRA, J. Manual de procedimientos para inventarios de carbono en ecosistemas forestales. Proyecto FONDEF/D98I1076. Valdivia: Universidad Austral de Chile, 2001.

SEGURA, M.; ANDRADE, H. ¿Cómo construir modelos alométricos de volumen, biomasa o carbono de especies leñosas perenes? Revista Agroforestería en las Américas, Costa Rica, n. 46, p. 89-96, 2008.

SIERRA, R. Propuesta preliminar de un sistema de clasificación de vegetación para el Ecuador Continental. Quito: INEFAN; GEFIBRD; EcoCiencia, 1999. p. 155-163.

UNGER, M.; HOMEIER, J.; LEUSCHNER, C. Efects of soil chemistry on tropical forest biomass and productivity at diferent elevations in the equatorial Andes. Journal Ecosytem Ecology, London, v. 170, p. 263-274, 2012.

URETA, M. Aporte de biomasa aérea de las especies arbóreas de la familia Myristicaceae en los bosques Amazónicos del Perú. Revista de Biología Tropical, Costa Rica, v. 63, n. 1, p. 263-273, 2015.

VALAREZO, C. Características, distribución, clasificación y capacidad de uso de los suelos en la región amazónica ecuatoriana-RAE. Ecuador: Universidad Nacional de Loja; Programa de Modernización de los Servicios Agropecuarios, 2004. 201 p. 
VALDÉS RAMÍREZ, M. Ensayo el cambio climático y el estado simbiótico de los árboles del bosque. Revista Mexicana de Ciencias Forestales, México, v. 2, n 5, p. 5-14, 2012.

VÁSQUEZ, A.; ARELLANO, H. Estructura biomasa aérea y carbono almacenado en los bosques del Sur y Noroccidente de Córdoba. En: RANGEL-CH., J. O. (Ed.). Colombia Diversidad Biótica XII. La región Caribe de Colombia. Bogotá: Universidad Nacional de Colombia, Instituto de Ciencias Naturales, 2012. p. 923-961.

WALPOLE, R.; MYERS, R. Probabilidad y estadística. 4 ed. México, McGraw-Hill. 1992. p 797.

YEPES-QUINTERO, A.; DUQUE-MONTOYA, Á. J.; NAVARRETE-ENCINALES, D.; PHILLIPSBERNAL, J.; CABRERA-MONTENEGRO, E.; CORRALES-OSORIO, A.; VARGAS-GALVIS, D. Estimación de las reservas y pérdidas de carbono por deforestación en los bosques del Departamento de Antioquia, Colombia. Actualidades Biológicas, v. 33, n 95, p. 193-208, 2011.

ZAMBRANO, A.; FRANQUIS, F.; INFANTE, A. Emisión y captura de carbono en los suelos en ecosistemas forestales. Revista Forestal Latinoamericana, Venezuela, v. 19-1, n. 35, p.11-20, 2004.

ZANNE, A. E. et al. Global wood density database. 2009. Disponible: 〈http://hdl.handle.net/10255/ dryad.235>. Acceso: 18 sept. 2015. 